\title{
Pancoast's syndrome associated with invasive aspergillosis
}

\author{
FG SIMPSON, M MORGAN, NJ COOKE \\ From the Departments of Respiratory Medicine and Haematology, General Infirmary, Leeds
}

The combination of lower brachial plexus root pain in the shoulder and arm and of ipsilateral Horner's syndrome was described by Pancoast in 1932 and is usually caused by a malignant tumour at the apex of the lung. We describe a case in which the syndrome occurred in an immunosuppressed patient and was due to invasive fungal infection.

\section{Case report}

A 34 year old woman was receiving induction chemotherapy with high dose cytosine arabinoside for relapsed acute myeloid leukaemia. During the neutropenic period she developed a fever, which responded to intravenous ceftazidime and amikacin. Blood cultures were sterile and antibiotic treatment was discontinued after nine days. One week later she developed an intermittent fever and some pain in the left shoulder. Over the next two days she complained of increasingly severe pain over the left scapula, which radiated along the medial aspect of the left upper arm. She had no cough or haemoptysis and a chest radiograph showed a small opacity at the left apex. Previous chest radiographs had been normal. Three days later she was noted to have a left Horner's syndrome with absence of sweating of the left hand. The neurological signs did not change over the next week but a chest radiograph showed an increase in the left apical opacity (fig 1). Myelography and computer assisted tomography of the root of the neck failed to show extrinsic root compression. The mass at the lung apex was demonstrated but no infiltration of the brachial plexus could be seen. A cytospin preparation of cerebrospinal fluid showed no evidence of central nervous system leukaemia. A percutaneous Trucut lung biopsy was performed via a suprascapular approach. Numerous fungal hyphae were seen on histological sections (fig 2) and identified as Aspergillus species morphologically, though there was no growth on culture. Repeated tests for serum precipitins against Aspergillus gave negative results. Treatment with intravenous amphotericin was started and after one week of treatment she was afebrile. The root pain resolved over the next 10 days but there was no improvement in the Horner's syndrome. Treatment continued for six weeks with almost complete resolution of the left apical opacity. A bone marrow aspirate showed that the leukaemia was in remission. Three months later she had a second relapse of her leukaemia, which was treated successfully without reactivation of the aspergillus infection. One year later she remains well with a persistent left Horner's syndrome.

Address for reprint requests: Dr FG Simpson, Department of Respiratory Medicine, General Infirmary, Leeds LS1 3EX.

Accepted 16 September 1985

\section{Discussion}

Non-malignant causes of Pancoast's syndrome are extremely rare. Pancoast himself described pulmonary tuberculosis presenting in this way. ${ }^{1}$ Hydatid cysts at the thoracic outlet ${ }^{2}$ or rupturing into the pleura ${ }^{3}$ may produce

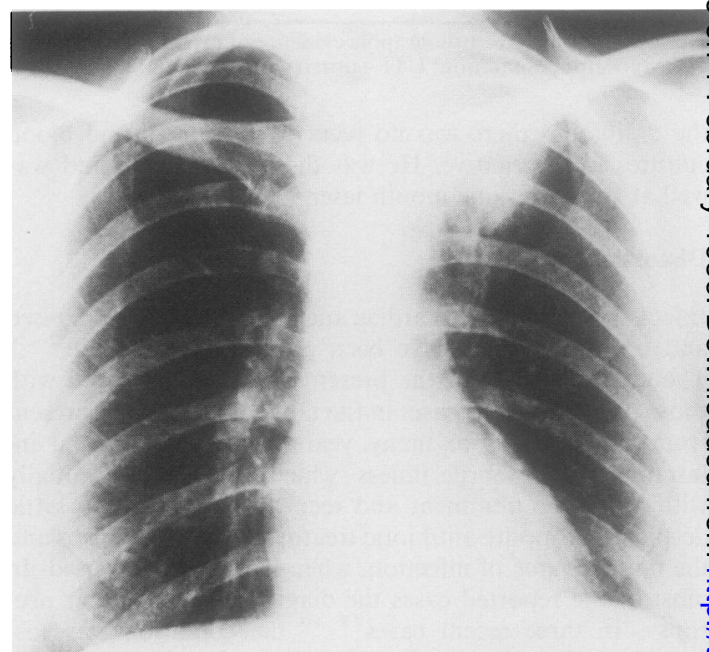

Fig 1 Chest radiograph two weeks after onset of Pancoast' syndrome.

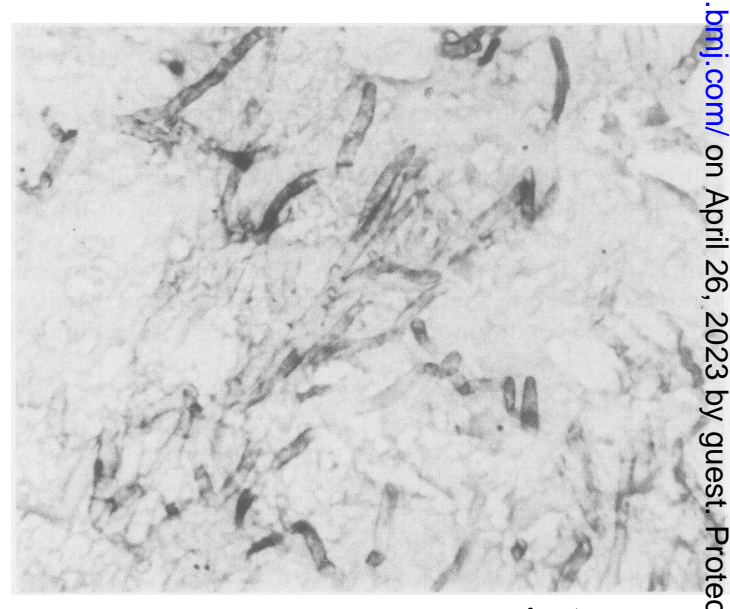

Fig 2 Percutaneous lung biopsy specimen showing numerou fungal hyphae. $(\times 250$.) 
the syndrome. There is a single report of a fungal infection by Allescheria boydii producing Pancoast's syndrome. ${ }^{4}$ Interestingly, this patient was also suffering from acute myeloid leukaemia and was further immunosuppressed by treatment with cytosine arabinoside. In this case, unlike the present one, there was no response to treatment with amphotericin B.

Although precise identification was impossible in our patient, the diagnosis was clearly fungal infection, and the organism was identified morphologically by mycologists as an Aspergillus species. The repeatedly negative results of tests for Aspergillus precipitins are explained by the profound immunosuppression. We have no explanation for the failure to grow the fungus in culture.

Despite the failure of computed tomography to demonstrate disease of the brachial plexus there can be little doubt that infiltration or inflammation of the lower part of the plexus caused the symptoms.

Fungal infection should be considered as a cause of Pan- coast's syndrome with lung shadowing in the immunosuppressed patient, as treatment of the infection may be successful.

We thank Dr JA Child for permission to report a case under his care, Dr EG Evans for mycological advice, and Mrs KA Marsden for secretarial help.

\section{References}

1 Pancoast HK. Superior pulmonary sulcus tumor. Tumor characterised by pain, Horner's syndrome, destruction of bone and atrophy of hand muscles. JAMA 1932;99:1391-6.

2 Stathatos C, Kontaxis NA, Zafiracopoulos P. Pancoast's syndrome due to hydatid cyst of the thoracic outlet. $J$ Thorac Cardiovasc Surg 1969;58:764-8.

3 Aletras H, Papaconstantinou C. Pancoast's syndrome following intrapleural rupture of a hepatic echinococcus cyst. Scand J Thorac Cardiovasc Surg 1982;16:283-7.

4 Winston DJ, Jordan MC, Rhodes J. Allescheria boydii infections in the immunosuppressed host. Am J Med 1977;63:830-5. 